\title{
Understanding the Interpersonal Space of Online Meetings: An Exploratory Study of "We-ness"
}

\author{
Victor Kaptelinin \\ Dept. of Informatics, Umeå University \\ victor.kaptelinin@umu.se \\ Christoffel Kuenen \\ Umeå Institute of Design, Umeå \\ University \\ christoffel.kuenen@umu.se
}

Karin Danielsson

Dept. of Informatics, Umeå University

karin.danielsson@umu.se

\author{
Niclas Kaiser \\ Dept. of Psychology, Umeå University \\ niclas.kaiser@umu.se
}

\author{
Maria Nordin \\ Dept. of Psychology, Umeå University \\ maria.nordin@umu.se
}

\begin{abstract}
The covid-19 pandemic has severely limited the possibility for people to meet physically, which forced many individuals and organizations to employ online meetings as their predominant mode of communication. A potential problem with the unprecedentedly central role of online meetings in a wide range of everyday activities is the disruption it may cause to intersubjective experiences, an intuitive mutual understanding of the participants and their thinking of themselves as a group, a "we". To address this problem, about half a year into the pandemic we conducted an exploratory study, in which the informants $(\mathrm{N}=36)$ completed a survey, comprising a set of Likert scales and open-ended questions focusing on "team spirit", moment-to-moment coordination, emotions, and the sense of presence in online and physical meetings. The results indicate that online meetings may present particular challenges regarding the experience of "we-ness", and different types of online meetings can be experienced differently. Implications of the results for further research are discussed.
\end{abstract}

\section{CCS CONCEPTS}

- Human-centered computing $\rightarrow$ Collaborative and social computing; Empirical studies in collaborative and social computing.

\section{KEYWORDS}

We-ness, Online meetings, Team spirit, Moment-to-moment coordination, Presence

\section{ACM Reference Format:}

Victor Kaptelinin, Karin Danielsson, Niclas Kaiser, Christoffel Kuenen, and Maria Nordin. 2021. Understanding the Interpersonal Space of Online Meetings: An Exploratory Study of "We-ness". In Companion Publication of the 2021 Conference on Computer Supported Cooperative Work and Social Computing (CSCW'21 Companion), October 23-27, 2021, Virtual Event, USA. ACM, New York, NY, USA, 5 pages. https://doi.org/10.1145/3462204.3481780

Publication rights licensed to ACM. ACM acknowledges that this contribution was authored or co-authored by an employee, contractor or affiliate of a national government. As such, the Government retains a nonexclusive, royalty-free right to publish or reproduce this article, or to allow others to do so, for Government purposes only. CSCW'21 Companion, October 23-27, 2021, Virtual Event, USA (c) 2021 Copyright held by the owner/author(s). Publication rights licensed to ACM. ACM ISBN 978-1-4503-8479-7/21/10 ..\$15.00

https://doi.org/10.1145/3462204.3481780

\section{INTRODUCTION}

Arguably, a key motivation for CSCW research on technologymediated communication has been creating a possibility for remote participants in online (or "virtual") meetings to experience themselves as a team, rather than a collection of information-exchanging individuals. However, the experience of "we-ness" in online meetings, that is, interpersonal connectedness in the flow of interaction [13], which supports the processes for participatory sensemaking and results in assuming a collective identity by the meeting participants, rarely became an explicit object of study.

A possible explanation is that technology-mediated meetings often have been combined with physical meetings. Physical meetings allow people to capitalize upon a rich diversity of sensory cues, which makes such meetings more suitable for team building, establishing personal connections, and immersive real-time collaboration [20]. While physical meetings may have significant advantages, their role in current work practices appears to decrease. A shift from physical to online meetings, which has been dramatically accelerated by the covid-19 pandemic [21, 22], is likely to continue even after the pandemic is over, and an increasing range of everyday activities is going to take place mostly, or even exclusively, online. Therefore, supporting interpersonal connectedness, "team experience", and the sense of being "we" in online meetings emerge as topical issues for CSCW research and practice.

This study aims to contribute to the research in these issues by investigating differences in we-ness in physical and online meetings, along with exploring how socially present the participants feel online. The study should be regarded as a pilot study, exploring whether the topic is fruitful to address from a cross-disciplinary perspective.

\section{RELATED RESEARCH}

In addition to research in CSCW and human-technology interaction in general, our study also capitalized on studies in social neuroscience and occupational health.

\subsection{CSCW research}

While CSCW research on virtual meetings has been mostly focusing on shared information and activity spaces (such as media spaces and distributed office environments $[2,10]$ ), a wide range of issues, relevant to understanding the feeling of being $a$ "we", experienced by remote participants, has also been explored. Some of the key 
issues include the relationship between "space" and "place" (the latter being a shared environment, filled with meaning, practice and a sense of presence [5]), integration of shared remote collaboration workspaces with interpersonal spaces [12], the role of gaze and gestures in computer-mediated communication [18], creating distributed work environments, in which collaborating parties all feel that they are present "here" rather than "there" [3], supporting the sharing of spatial properties of action in video conferences [19], and providing design solutions for more inclusive hybrid meetings [9]. The studies found that closed-knit coordination is important $[1,2,8,10,12,18]$, that remote participants may feel excluded [20], and that participants' feeling and emotions (e.g., empathy [18]), as well as the sense of presence [3] should be taken into consideration. At the same time to the best of our knowledge, participants' experience of "we-ness" has not been a key and explicit object of systematic analysis on CSCW research.

\section{2 "We-ness" in social neuroscience and occupational health research}

Little is known about what affects we-ness, but in relation to onlinemeetings it has been shown that even a short delay of a second strongly influences conversational flow and emotional comfort in a negative way [14] supporting the assumption that being in the same time-space is key in forming positive group interaction. Moreover, eye contact is important in social interaction [7, 11]. Thus, skewed camera positions in video meetings may have negative effects on social reciprocity. A helpful way of thinking when putting biological systems in relation to we-ness in mediated meetings is social breathing [13]. This latent construct behind the multitude of biological systems, summarizes interdisciplinary findings that engagement in - and the maintenance of - social systems is a multi-facetted process that involves the whole brain-body-system. The specifics in how online-communication influences the real relationships is to our knowledge still a highly understudied question.

Work is a prioritized arena for health promotion [4,23] and Lee [15] argues that the concept of we-ness at work is important for both performance and productivity. Reliable relations and social support are key factors in a healthy work environment, inhibiting damaging stress responses [6]. Therefore, the questions in our study refer to work meetings.

\section{A TENTATIVE FRAMEWORK}

For the purposes of the present study, we developed a framework, tentatively identifying key aspects of the experience of "we-ness" in online meetings. As discussed above, the research conducted in $\mathrm{CSCW}$, social neuroscience and occupational health has identified a range of relevant issues, including: coordination, participants' feeling and concerns, and the sense of presence. The framework we developed reflects the above issues, as well as the user-system interaction. The main components of the framework, shown in Figure 1, are, accordingly, (A) the overall team experience, comprising: (B) the moment-to-moment coordination of a participant's engagement in group communication; (C) user's feelings, emotions and concerns during an online meeting; (D) user's sense of presence in the "social space" of the online meeting, rather than their immediate physical

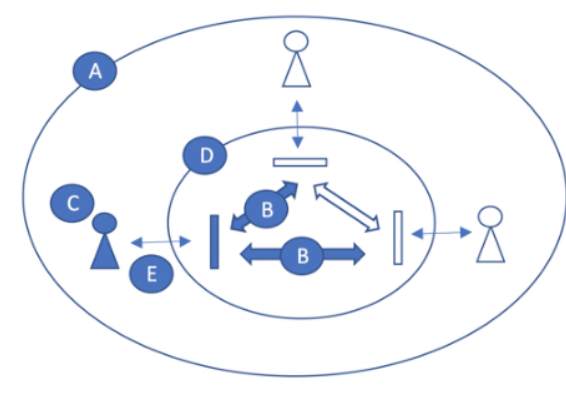

Figure 1: Aspects of user experience of online meetings, addressed in the study: A - overall experience of being a part of a team, B - moment-to-moment coordination of a meeting, $C$ - user's emotions and concerns, D - the feeling of presence, $\mathrm{E}$ - interaction between the user and the technology.

environment; and (E) interaction between the user and technology (such as Zoom, MS Teams, or Google Meet).

\section{METHOD}

We developed and administered a survey that comprised two types of questions, collectively covering components A-E of the above framework. Questions of the first type were asked about both physical and online meetings. They dealt with team experience (feeling a part of a team or as an outsider), coordination (understanding other participants and their expectations, as well as how difficult it is to find an appropriate time for one's contribution), and feelings/ emotions (enjoying a meeting, being stressed, being concerned about how one looks). Questions of the second type only dealt with virtual meetings, inquiring about: (a) forming a team with new people vs. people one met before, physically or online, (b) concerns about how one's environment looks, (c) experiencing presence in one's physical environment or in the "social space" of the meeting, and (d) assessing the interaction with technology (how distractive it is and what functionality is missing). The questions were rated on a Likert scale from 1 (disagree) to 7 (agree). In addition, all questions had space for reflections in free text. The survey was posted on Facebook in October of 2020 and was available for a month.

Means and standard deviations were calculated and one sample t-tests were conducted for comparing the experiences in physical and online meetings. The free text answers were analyzed by a simplified qualitative text analysis [17].

\section{RESULTS}

In our online survey, 36 people participated. Out of these, $69 \%$ were women, $56 \%$ were 41 to 50 years and $25 \%$ were between 51 and 60 years. One participant was from Norway, two did not state their country of residence and the rest were from Sweden. The participants' occupations were mixed but all required university degrees and included, for example, project managers, researchers, court lawyers, programmers and students. The participants used mostly Zoom and Teams and varied largely in experience with virtual meetings $($ mean $=34.7$ months, $\mathrm{sd}=51.7$, median $=8$ ). 


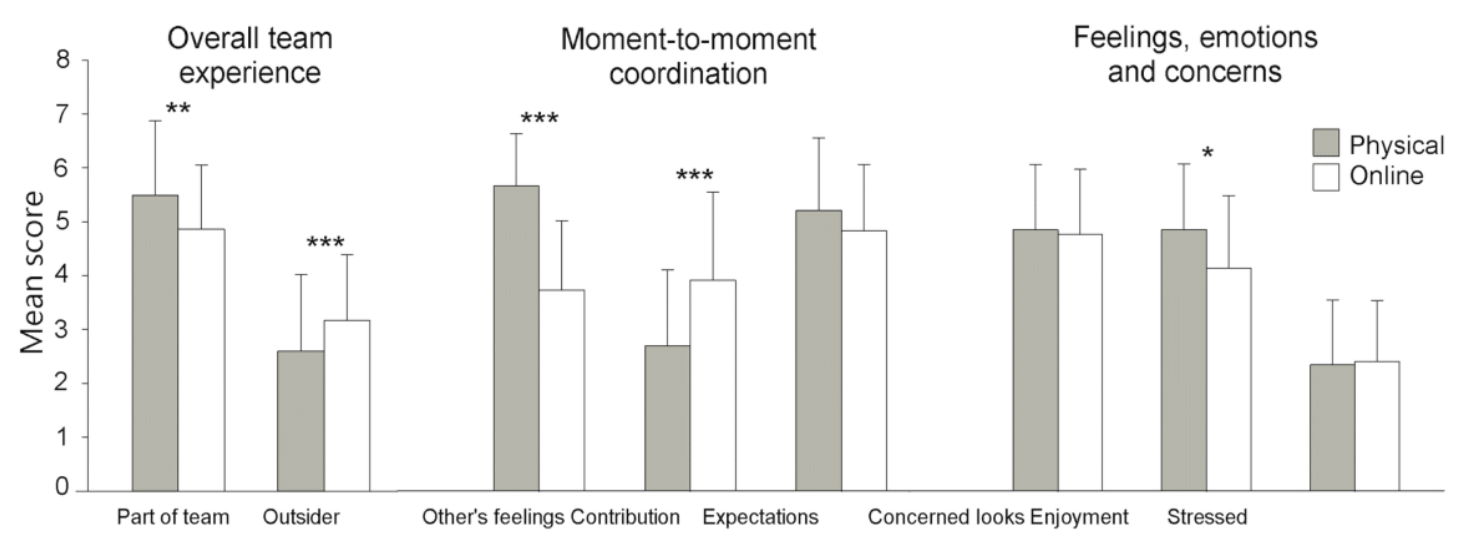

Figure 2: Mean values of survey questions rated for in physical and online meetings $\left({ }^{*} \mathbf{p}<0.05,{ }^{* *} \mathbf{p}<0.01,{ }^{* * *} \mathrm{p}<0.001\right)$.

\subsection{A. Overall team experience}

One-sample t-tests for overall team experience show a statistically significant difference in being part of a team and feeling as an outsider in favor of physical meetings (Cohen's ds $=0.33$ and 0.43 ). Free text answers nuanced the results describing the importance of (i) the purpose of the meeting, (ii) if the participants knew each other and (iii) number of participants as important factors. Online meetings were considered more anonymous, more task-oriented, not always aimed for team building and easier to hide in even if they also were easier to "kidnap" since two people could maintain a dialogue simultaneous to shutting the others out.

\subsection{B. Moment to moment coordination}

Regarding the experience of moment-to-moment coordination, understanding others' feelings and difficulties making contributions in a meeting differed significantly between the physical and online meetings (Cohen's ds $=1.57,0.69$, see middle part of Figure 2). However, there was no significant difference in type of meeting when it came to understanding what was expected (Cohen's $\mathrm{d}=0.32$ ). Results from text data revealed the importance of cameras being turned on for having a chance to understand others' feelings. Difficulty in spotting each other's body language, making pauses unnaturally long, as well as delays in sound, also made it more difficult to understand each other in online meetings. However, it was clearly stated that clarity on what was expected of the participants was important regardless of type of meeting.

\subsection{Feelings, emotions and concerns}

As for feelings, emotions and concerns, the concern about looks was rated the same regardless of type of meeting. Physical meetings were considered significantly more enjoyable and less stressful than online meetings (Cohen's ds $=0.07,0.50$ and 0.05 ). Analyses of text data showed that some were more self-aware in online meetings since they could see themselves, whereas others were equally concerned regardless of type of meeting. The most important factor for enjoying the meeting was the purpose of it and whether it was well organized or not. Most participants responded that there was no difference in the experience of stressed feelings in physical and online meetings. However, someone pointed out that it is easier to hide online.

\subsection{Presence in the "social space" of the online meeting}

When it came to the experience of feeling present in online meetings, the participants scored on average $4.71(\mathrm{sd}=1.47)$ on the question feeling present in the environment and $4.44(\mathrm{sd}=1.38)$ on the question on feeling present in the virtual "social space" of interaction with other people. However, text data revealed that these questions were difficult to understand for the participants.

\subsection{E. Human-technology interaction}

The survey questions on technology working smoothly and not distracting me from interaction with other people had a mean rating of 4.71 ( $\mathrm{sd}=1.45)$, and the question on the technologies I use do not have certain functions which I would like to have a mean score of $3.88(\mathrm{sd}=1.77)$. The participants stated that they were worried about the technologies breaking down which was considered stressful. Moreover, the functions in the different apps were said to be a bit confusing. The participants wished for functions similar to a white board when sharing screens. Also, breakout rooms were appreciated and wished for in all apps.

\section{DISCUSSION}

The survey was conducted more than six months into the covid-19 pandemic and all participants had types of jobs that had been transferred online. Therefore, all participants had extensive, albeit varied, experience with work-related online meetings. An indicator of this experience is that the use of technology was considered smooth and unproblematic in general. Still, the perception of online meetings was generally less favourable than that of physical meetings. The participants gave online meetings significantly lower scores on the scales of team experience, understanding others' feelings, and the 
ease of making a contribution to a conversation. Moreover, they enjoyed physical meetings more.

The findings that, despite an extensive practice, the participants perceived online meetings as an inferior format in terms of team experience, coordination, and emotions. In addition, the fact that the participants found it more difficult to feel like a team and understand others' feelings in online meetings, agrees with contemporary knowledge about the biological social system being both time-lag sensitive [14] and depending on shared visuality $[7,16]$. Furthermore, the support for we-ness is an embodied system [13] is naturally limited in online communication. Therefore, supporting embodiment may be a way to address the limitations of online meetings.

Somewhat surprisingly, we did not find a significant difference between physical and online meetings in terms of overall stress. There can be different possible explanations of this result. For instance, the level of stress may depend on whether or not the participants know each other beforehand, which may be one component worth taking into consideration when choosing an online meeting. Another explanation, consistent with participants' free text responses, is that some aspects of online meetings (e.g., technological breakdowns) increase participants' stress, while other aspects (e.g., the possibility to "hide") decrease it.

A key direction for future research is comparative analysis of different types of meetings. As many participants indicated in their comments, the overall assessment of a meeting and the experience of its particular aspects, strongly depend on the purpose and structure of a meeting, irrespective of whether the meeting is physical or virtual. Future research should also explore the effect of age, gender, occupation, and other social categories on the experience of "we-ness" in online meetings.

Finally, the study should be considered as exploratory, which makes the results tentative and the limitations of the study important to consider. First of all, the study includes a small number of participants being conveniently sampled from social media, giving a highly selected sample. However, the sample was rather heterogeneous with regard to age, sex, occupation and experience with online meetings. The questionnaire did not include any validated questions and our framework has not been empirically tested before. However, the participants responded properly to all questions except for the one on being present in the "social space", and there were no ceilings or floor effects in the responses. The text answers were valuable as they offered more nuance to the questions asked. These insights can be used for developing the questionnaire further.

\section{CONCLUSION}

The current massive transition from physical to online meetings may mean that complete "team lifecycles", from emergence to dissipation, will increasingly involve little or no physical meetings. This trend presents new challenges for CSCW research and society in general, and by presenting a tentative framework for analyzing we-ness, as well as results of an exploratory study, this paper aims to contribute by addressing these challenges.

\section{REFERENCES}

[1] Kevin Baker, Saul Greenberg, and Carl Gutwin. 2002. Empirical development of a heuristic evaluation methodology for shared workspace groupware. In Proceedings of the 2002 ACM conference on Computer supported cooperative work
(CSCW '02). Association for Computing Machinery, New York, NY, USA, 96-105. https://doi.org/10.1145/587078.587093

[2] Sara A. Bly, Steve R. Harrison, and Susan Irwin. 1993. Media spaces: bringing people together in a video, audio, and computing environment. Commun. ACM 36, 1 (Jan. 1993), 28-46. https://doi.org/10.1145/151233.151235

[3] Michael Broughton, Jeni Paay, Jesper Kjeldskov, Kenton O’Hara, Jane Li, Matthew Phillips, and Markus Rittenbruch. 2009. Being here: designing for distributed hands-on collaboration in blended interaction spaces. In Proceedings of the 21st Annual Conference of the Australian Computer-Human Interaction Special Interest Group: Design: Open 24/7 (OZCHI '09). Association for Computing Machinery, New York, NY, USA, 73-80. https://doi.org/10.1145/1738826.1738839

[4] Pamela Brown, Kimberly Fraser, Carol A. Wong, Melanie Muise and Greta Cummings. 2013. Factors influencing intentions to stay and retention of nurse managers: a systematic review. Journal of Nursing Management 21(3), 459-472. https://doi.org/10.1111/j.1365-2834.2012.01352.x

[5] Paul Dourish, 2006. Re-space-ing place: " place" and" space" ten years on. In Proceedings of the 2006 20th anniversary conference on Computer supported cooperative work, November 2006 (pp. 299-308). https://doi.org/10.1145/1180875. 1180921

[6] Naomi I. Eisenberger, 2013. Social ties and health: a social neuroscience perspective. Current opinion in neurobiology, 23(3), 407-413. https://doi.org/10.1016/j. conb.2013.01.006

[7] Nathan J. Emery. 2000. The eyes have it: the neuroethology, function and evolution of social gaze. Neuroscience and Biobehavioral Reviews, 24(6), 581-604. https://doi.org/10.1016/S0149-7634(00)00025-7

[8] Naomi T. Fitter, Luke Rush, Elizabeth Cha, Thomas Groechel, Maja J Matarić, and Leila Takayama. 2020. Closeness is Key over Long Distances: Effects of Interpersonal Closeness on Telepresence Experience. In Proceedings of the 2020 ACM/IEEE International Conference on Human-Robot Interaction (HRI '20), March 23-26, 2020, Cambridge, United Kingdom. ACM, New York, NY, USA, 9 pages. https://doi.org/10.1145/3319502.3374785

[9] Jens Emil Grønbæk, Banu Saatçi, Carla F. Griggio, and Clemens Nylandsted Klokmose. 2021. MirrorBlender: Supporting Hybrid Meetings with a Malleable Video-Conferencing System. In Proceedings of the 2021 CHI Conference on Human Factors in Computing Systems (CHI '21). Association for Computing Machinery, New York, NY, USA, Article 451,1-13. https://doi.org/10.1145/3411764. 3445698

[10] Christian Heath and Paul Luff. 1991. Disembodied conduct: communication through video in a multi-media office environment. In Proceedings of the SIGCHI Conference on Human Factors in Computing Systems (CHI '91). Association for Computing Machinery, New York, NY, USA, 99-103. https://doi.org/10.1145/ 108844.108859

[11] Jonne O. Hietanen, Mikko J. Peltola and Jari K. Hietanen, J. K. 2020. Psychophysiological responses to eye contact in a live interaction and in video call. Psychophysiology, 57(6). https://doi.org/10.1111/psyp.13587

[12] Hiroshi Ishii, Minoru Kobayashi, and Jonathan Grudin. 1993. Integration of interpersonal space and shared workspace: ClearBoard design and experiments. ACM Trans. Inf. Syst. 11, 4 (Oct. 1993), 349-375. https://doi.org/10.1145/159764. 159762

[13] Niclas Kaiser and Emily Butler. 2021. Introducing Social Breathing: A Model of Engaging in Relational Systems. Frontiers in Psychology, 12. https://doi.org/10. 3389/fpsyg.2021.571298

[14] Namkje Koudenburg, Tom Postmes, and Ernestine H. Gordijn. 2011. Disrupting the Flow: How Brief Silences in Group Conversations Affect Social Needs. Journal of Experimental Social Psychology 47, nr 2 (01 mars 2011): 512-15. https://doi. org/10.1016/j.jesp.2010.12.006.

[15] Hyunjung Lee \& Stephen K. Markham, 2013. We-ness, knowledge sharing, and performance in New Product Development Teams. In The PDMA handbook of new product development, 3rd edition. Ed K.B. Kahn, pp 181-194. New Jersey, USA.

[16] Charles Lenay, John Stewart, Marieke Rohde and Amal A. Amar. 2011. You never fail to surprise me: The hallmark of the Other: Experimental study and simulations of perceptual crossing. Interaction Studies, 12(3), 373-396. https: //doi.org/10.1075/is.12.3.01len

[17] Kirsti Malterud. Systematic text condensation: A strategy for qualitative analysis. Scandinavian Journal of Public Health 40 (december 2012): 795-805. https://doi. org/10.1177/1403494812465030.

[18] David T. Nguyen and John Canny. 2009. More than face-to-face: empathy effects of video framing. In Proceedings of the SIGCHI Conference on Human Factors in Computing Systems (CHI '09). Association for Computing Machinery, New York, NY, USA, 423-432. https://doi.org/10.1145/1518701.1518770

[19] Kenton O'Hara, Jesper Kjeldskov, and Jeni Paay. 2011. Blended interaction spaces for distributed team collaboration. ACM Trans. Comput.-Hum. Interact. 18, 1, Article 3 (April 2011), 28 pages. https://doi.org/10.1145/1959022.1959025

[20] Parviz F. Rad and Ginger Lewin. 2003. Achieving project management success using virtual teams. J. Ross Publishing: Boca Raton, FL., USA. 
[21] Daniel Russell, Carman Neustaedter, John Tang, Tejinder Judge, and Gary Olson 2021. Videoconferencing in the Age of COVID: How Well Has It Worked Out? Extended Abstracts of the $2021 \mathrm{CHI}$ Conference on Human Factors in Computing Systems. Association for Computing Machinery, New York, NY, USA, Article 151, 1-2. https://doi.org/10.1145/3411763.3450398
[22] World Health Organization, WHO, retrieved June 4, 2021 from https://www.who. int/emergencies/diseases/novel-coronavirus- 2019

[23] WHO 2021 The workplace: A priority setting for health promotion. Retrieved June 7, 2021 from https://www.who.int/occupational_health/topics/workplace/en/ 